\title{
La ecología cultural política y el estudio de regiones en México
}

\author{
Political Cultural Ecology and the Study \\ of Regions in Mexico
}

\author{
Andrés Fábregas Puig \\ Universidad Intercultural de Chiapas. México
}

\section{RESUMEN}

El artículo muestra por qué la ecología cultural, entendida como un método para indagar en las relaciones entre el medio ambiente y los procesos culturales ha arraigado en una generación de antropólogos mexicanos como consecuencia del magisterio de Ángel Palerm y Eric Wolf. La aplicación práctica de las teorías de Steward a los procesos de formación regional mexicana vinculados a usos políticos de tecnologías hidráulicas como parte, tanto de estrategias culturales de adaptación como de control político del agua en el proceso de producción de alimentos, ha puesto de manifiesto que la ecología cultural, entendida como manejo cultural de entornos naturales, al igual que la economía, es diseñada y manejada políticamente en el contexto de sociedades profundamente desiguales con consecuencias maladaptantes.

Palabras clave: Ecología cultural; Palerm; Sociedad Hidráulica; México; Chiapas.

\section{SUMMARY}

This paper shows why Cultural Ecology, understood as a method to explore the relationships between the environment and cultural processes, has deeply influenced a whole generation of Mexican anthropologists as a result of the teachings of Ángel Palerm and Eric Wolf. The practical application of Steward's theories to the processes of Mexican regional formation, linked to the use of hydraulic technologies as part of both adaptative cultural strategies and the political control of water in the process of food production, shows that Cultural Ecology, understood as cultural management of natural environments, as it happens with Economy, is designed and politically manipulated in the context of deeply unequal societies with unadaptative consequences.

Key Words: Cultural Ecology; Palerm; Hydraulic Society; México; Chiapas. 
La ecología cultural como un método desarrollado por los antropólogos fue contextualizado teóricamente en México por Ángel Palerm y Eric Wolf. En efecto, en varios textos Palerm y Wolf (1954-1955) usaron el enfoque de la ecología cultural para analizar la etnohistoria de los Valles Centrales mexicanos y más precisamente, lo que ambos llamaron el "área del Acolhuacan" (Texcoco, Estado de México). En trabajos anteriores a los de Palerm y Wolf, como en el caso del antropólogo mexicano Manuel Gamio (1922) o en el del antropogéografo Jorge A. Vivó (1951), se señaló la importancia de comprender el medio ambiente natural en el análisis de una Cultura o de una Sociedad. En sus textos de la década de 1950-1960, Gonzalo Aguirre Beltrán (1967) llamaba la atención hacia la importancia del medio ambiente y la interrelación que los grupos humanos establecen con él, para entender la formación y la configuración de regiones. Palerm y Wolf (1972) reconocieron su descendencia teórica de la escuela antropológica elaborada por Julian Steward (1955) que aplicó el método de la ecología cultural uniéndolo a las explicaciones evolucionistas multilineales. La orientación ecológica cultural fue enlazada por Wolf (1959) y Palerm (1980) al marxismo, aunque ambos lo hicieron de manera diferente. En el caso de Palerm, su influencia se inicia en México en el año de 1966 a través del curso intensivo de teoría etnológica que dictó en la Escuela Nacional de Antropología e Historia, ante un grupo de estudiantes que por vez primera escuchaban una exposición sistemática de teorías antropológicas. Además de revisar las escuelas del momento, Palerm se extendió en una reflexión acerca del evolucionismo y de las relaciones que guardó desde el siglo XIX con los planteamientos que venía elaborando Karl Marx (Palerm 1967). Más aún, a cierta distancia de que Lawrence Krader diese a conocer en 1972 la existencia y el contenido de los Cuadernos de Notas Etnológicas de Marx, Ángel Palerm advirtió la influencia del evolucionismo antropológico en el pensamiento de Marx. Al respecto, Palerm destacaba la importancia del medio ambiente natural, tanto en el planteamiento evolucionista, como en el de Marx. Esta asociación quedaría mejor demostrada en los textos escritos por Karl W. Wittfogel (1957), aun con las reticencias de éste para reconocerlo. Sobre todo, los conceptos de "sociedad oriental" y "despotismo oriental" que maneja Wittfogel, sin mencionar la importancia de las obras hidráulicas en la conformación del poder político que Marx ya había resaltado, y que fueron reintroducidos a la antropología en México por Ángel Palerm.

Después del curso de introducción a la teoría etnológica de 1966 y pasado el movimiento estudiantil de 1968, Palerm, que junto con su esposa Carmen Viqueira, habían replanteado la enseñanza de la antropología en la Universidad Iberoamericana de la Ciudad de México, además de que ambos fundasen la Casa José de Acosta en el poblado de Tepetlaoztoc (Esta- 
do de México), inició una serie de seminarios en la Escuela de Graduados de la Universidad Iberoamericana, insistiendo en la aplicación del método de la ecología cultural y la explicación evolucionista multilineal. En esos años de 1969 a 1971, se discutió la antología de Cohen (1974), Man in Adaptation que contiene el texto del propio Cohen, "Culture as Adaptation", leído y aplicado intensamente en los estudios antropológicos emprendidos en México por los alumnos de Palerm. Esta fue una primera contextualización teórica que Palerm presentó a sus alumnos, destacando que la ecología cultural ha sido desarrollada a partir de los textos de Julian Steward, quien a su vez, enfatizó la multilinealidad de la evolución. A esta orientación teórica le interesa explicar la dinámica de una Evolución General que se elabora a partir de lo que Sahlins denomina "evoluciones particulares" (Sahlins y Service 1960), las que, a su vez, son puestas en marcha por adaptaciones humanas concretas. Son estas el objeto de estudio de la antropología. Ángel Palerm ilustraba lo anterior discutiendo la obra de Wittfogel y el concepto de "sociedad hidráulica". Esta discusión le facilitaba introducir los planteamientos de Marx y asimilarlos a la explicación evolucionista multilineal. Hacia 1852-1853, Karl Marx (1968a, 1968b) escribió artículos para el New York Daily Tribune en los que expuso su visión del Oriente, revelando su concepción sobre el colonialismo y su relación con la expansión del capitalismo, planteamiento que después enfatizó Lawrence Krader (1973). En esos artículos sobre el Oriente, Marx señaló con claridad la conexión entre las condiciones del medio ambiente y las características del "poder despótico" del Estado. Son justo los planteamientos retomados por Wittfogel y desarrollados en su Oriental Despotism. Son, así mismo, los enfoques que Ángel Palerm introduce para analizar el Valle de México, como lo escribió en sus textos de principios de los años 1950 y posteriores (1952, 1955, 1990).

En 1971, invitados por Palerm, los antropólogos evolucionistas Robert A. Manners y David Kaplan, desarrollaron un seminario teórico en la Escuela de Graduados de la Universidad Iberoamericana, utilizando como texto básico para la discusión una antología preparada por ellos mismos, Theory in Antropology (1970). Manners y Kaplan insistieron en la aplicación del método de la ecología cultural y su asociación con el evolucionismo multilineal, apegándose a los enfoques de Julian Steward. Introdujeron el análisis del libro compilado por este último, The People of Puerto Rico (Steward 1956), enfatizando la discusión de los conceptos de "niveles de articulación" y de "niveles de integración", propuestos por el propio Steward. Kaplan había participado en el grupo de antropólogos que estudiaron Puerto Rico bajo la guía de Steward, esto es, entre otros, Sydney Mintz, Eric Wolf, Jhon Murra, quien fungió como director del trabajo de campo en ese grupo y quien aplicó con resultados espléndidos el método de la ecología cultural en sus estu- 
dios de la etnohistoria de Perú (1975). Es a esta escuela antropológica a la que perteneció Palerm, quien, junto con Eric Wolf, insistió en los enlaces de la antropología con el marxismo.

En el caso mexicano, Ángel Palerm relacionó la discusión marxista acerca del Modo Asiático de Producción (MAP) con el planteamiento de Wittfogel en torno a la sociedad oriental (hidráulica) y el método de la ecología cultural, para aplicarlos al estudio etnohistórico del Valle de México. Aquí estaba en ciernes el planteamiento de una ecología cultural política asociada a la economía política, asociación explicitada posteriormente en los trabajos de Brigitte Boehm (1986, 2005) Andrés Fábregas (1986, 2003), Tomé y Fábregas (1999) y otros. En términos del MAP, Palerm afirmó la "evolución múltiple" de la Cultura, rechazando el esquema proveniente del marxismo de la Academia de Ciencias de la Unión Soviética, que insistía en una visión lineal de la Historia, la tan conocida secuencia, comunismo primitivoesclavismo-feudalismo-capitalismo-socialismo-comunismo. Hacia 1972, la crítica a la secuencia anterior como una línea histórica universal se amplió con la presencia de Lawrence Krader en el entonces Centro de Investigaciones Superiores del Instituto de Antropología e Historia (CIS-INAH), dirigido por Ángel Palerm. Las aportaciones de Krader alcanzaron la discusión entablada entre los marxistas en torno a la cuestión del Modo Asiático de Producción y su relación con la llamada "sociedad hidráulica". De inicio, Krader (1975), rechazó la adjetivación "sociedad hidráulica" argumentado que no es la tecnología la que otorga su naturaleza a la sociedad. En todo caso, la tecnología hidráulica es parte de una estrategia de adaptación que implica como factor básico al control del agua en el proceso de producción de alimentos. Más aún, Krader, asume críticamente el concepto de Modo Asiático de Producción y lo rechaza porque implica aceptar una categoría geográfica determinando la naturaleza de un modo de producción. En rigor, el término es un desliz del propio Marx que en este caso, no es fiel a sus propios planteamientos. En efecto y según Marx, ¿qué define a un modo de producción? El propio Marx lo señaló con claridad: la condición concreta del trabajo. En otras palabras, son las relaciones sociales que enmarcan al trabajo las que definen a un modo de producción. En consecuencia, no existen modos de producción "europeos", "americanos" o "asiáticos", sino feudales, esclavistas o capitalistas. La estrategia de adaptación de una determinada ecología cultural es puesta en marcha a través del trabajo y son las relaciones en torno a este las que otorgan las características definitorias de un modo de producción. La diferencia importante entre Wittfogel y Krader radica en la manera de plantear la importancia de las relaciones de trabajo en la configuración de un modo de producción y la puesta en práctica de una estrategia de adaptación. Tanto Marx, como posteriormente Wittfogel y 
Krader, notaron la importancia de la comunidad en el Oriente en general y de sus relaciones con el Estado. Marx habló, ciertamente, de un "despotismo oriental" hecho posible por la configuración de las comunidades. Wittfogel siguió este camino añadiendo que existe una vinculación directa entre propiedad comunal y Estado despótico. Ni Marx — jasombroso! — ni Wittfogel analizaron las relaciones concretas de trabajo en el llamado Modo Asiático de Producción. En Wittfogel, son las condiciones de la estrategia de adaptación (la ecología cultural) las que permiten la emergencia y consolidación de "un Estado más fuerte que la sociedad" (Wittfogel 1966), apoyado en la propiedad corporada. En Marx, es la autosuficiencia de la comunidad, su incapacidad de transformación, lo que permite el establecimiento del Estado Despótico. Krader, retomando el criterio marxista de analizar las condiciones del trabajo, diseccionó las condiciones del mismo en el Oriente y encuentra que el trabajo está atado a la comunidad. Es esta atadura la que impide un "mercado libre de trabajo" y obliga al productor directo a ser mediado en sus relaciones de producción por la comunidad misma, con independencia de la estrategia de adaptación operante. La comunidad como órgano corporado es la que se relaciona con el Estado a través de la renta de la tierra en forma de impuesto (el tributo). No es el productor directo quien paga una renta al Estado para tener acceso al medio de producción estratégico, la tierra, sino la comunidad. Esa es la característica central del Modo de Producción, que no es asiático, sino Comunal Corporado. Marx lo notó, pero, ¿por qué no lo aclaró? Wittfogel optó por el determinismo tecnológico en un medio ambiente que exige el control del agua para hacer posible la producción. Más todavía, Wittfogel estableció una reciprocidad entre propiedad colectiva - sin analizar el contexto- y un Estado despótico, lo que le valió un permanente pleito con los marxistas.

En este contexto teórico, Palerm se situó más al lado de Wittfogel que de Krader y estableció la existencia de la "Sociedad Hidráulica" en el Valle del Anáhuac. Lo cierto es que ello abrió otras posibilidades de análisis en Mesoamérica, más allá del difusionismo de Paul Kirchhoff y de las explicaciones de orden religiosa implicadas en el concepto de "centro ceremonial" que se aplicaba a las ciudades. Palerm insistió en la existencia de ciudades en Mesoamérica, con características diferentes a las europeas. Son estas ciudades mesoamericanas, centros de poder, de intercambio económico y de control ideológico vía la religión. Lo importante para la etnohistoria del Anáhuac, es que hablándose de un modo u otro de producción, en Mesoamérica como en Oriente, la cuestión es la existencia y operación de una ecología cultural políticamente decidida y dirigida. Es justo lo que demostró Brigitte Bóehm en su libro sobre la formación del Estado en el México prehispánico (1986). Mesoamérica —como "área cultural”- conoció el es- 
tablecimiento del Estado y de una burocracia asociada que manejó los asuntos públicos. El propio Wittfogel incluyó a Mesoamérica como un "área marginal" del "despotismo oriental", debido a la importancia de las obras públicas de control del agua que existieron en el Valle del Anáhuac, concebidas y ejecutadas desde el Estado. Esta visión de Palerm, expuesta desde 1952, planteó también la pregunta de cómo se sostuvieron las ciudades en el territorio de los Mayas, hacia el Sur de México y de Centroamérica actuales. La arqueología más reciente, confirma la existencia entre los Mayas de un sistema político cercano a la organización en Ciudades-Estado, rivales entre sí, pero con capacidad para movilizar a grandes contingentes para la construcción de las obras públicas: terrazas de cultivo, canales subterráneos y las propias ciudades, entre otras. Todo ello, vino a demostrar la inexistencia del feudalismo en la antigüedad del Nuevo Mundo y a confirmar las tesis centrales del evolucionismo multilineal acerca de la variedad de la Evolución y la tarea de la antropología, en sus diferentes enfoques, de analizar en concreto cada adaptación y línea evolutiva.

No sólo en el campo de la etnohistoria surtió resultados positivos la conjugación del método de la ecología cultural con el marxismo. Fue esta orientación la que se aplicó — a instancias de Palerm - en el análisis contemporáneo del Valle de Texcoco y alrededores, más o menos, el territorio abarcado por el antiguo Acolhuacan estudiado por Palerm y Wolf. Los estudiantes del Departamento de Antropología Social de la Universidad Iberoamericana en los años de 1969 a 1975 desarrollaron un intenso trabajo de etnografía de las ecologías culturales de la región. Por ejemplo, el trabajo de Lucila Gómez Sahagún en San Nicolás Tlaixpan, dio como resultado una detallada etnografía del cultivo de las flores y de la agricultura de las huertas, lo que posibilitó la caracterización de la economía campesina de la región. La ganadería también fue analizada desde una perspectiva ecológica cultural, es decir, como una estrategia de adaptación diferente a la agricultura, basada en el cultivo del maíz en el contexto de una economía de autoconsumo. El centro nervioso de estos análisis fue la Casa José de Acosta en el pueblo de Tepetlaoztoc, la "Estación de Campo" del Departamento de Antropología de la Universidad Iberoamericana. En dicha Casa sucedieron largos diálogos entre estudiantes y antropólogos como William Sanders, Eric Wolf, Bárbara Price, Arturo Warman, Guillermo Bonfil o Ángel Palerm y Carmen Viqueira. Fue en esta Casa en donde conversaron Ángel Palerm y Efraín Hernández Xolocotzin, "Xolo", diálogo que enlazó a la antropología y la agronomía. Xolocotzin y Palerm discutieron cómo se transformaron las plantas en una parte de la Cultura y las diferentes ecologías culturales a que ello dio lugar. No sólo se discutió la importancia del tratamiento agronómico sino del manejo cultural de las plantas, la implicación de ello en la ge- 
neración de conocimiento y en lo que actualmente llamamos "diálogo de saberes". Convertida en centro de la pesquisa antropológica, desde la Casa José de Acosta se exploró la "Frontera Chichimeca", discutiéndose en el terreno mismo aspectos complejos de la etnohistoria de los Valles Centrales de México. El examen detallado de los cultivos, tanto en el valle como en el somontano, sin descuidar los sistemas de terrazas, fue posible por la aplicación del método de la ecología cultural y la búsqueda de explicaciones de las transformaciones en las estrategias de adaptación regionales, cómo se articularon y cómo se integraron en un ámbito regional. Fue un momento en el que se logró reivindicar la etnografía como ejercicio por excelencia de la antropología y una de las bases en el establecimiento de regiones en México. Más aún, a través de la búsqueda de los patrones de adaptación de la región, fue posible enlazar el pasado con el presente y comprender la continuidad de una tecnología que probó con creces su eficacia para producir alimentos en grandes cantidades y sostener a ciudades del tamaño de Texcoco o la Gran Tenochtitlán.

Al iniciarse la década de 1970 en México, el Estado Nacional se vio confrontado, entre otros grupos, por campesinos que reclamaban hacer efectivo su derecho al reparto agrario, anunciado y prometido por la Revolución de 1910. Al mismo tiempo, el crecimiento de las ciudades y de la planta industrial invadiendo los terrenos ejidales, exigía una mayor producción de energía eléctrica. Para hacer frente a este nuevo contexto, el Estado Nacional decidió inducir el poblamiento de la selva oriental de Chiapas (la llamada "Selva Lacandona"), además de construir una serie de hidroeléctricas en el cauce del río Grijalva. La inducción del poblamiento de la selva chiapaneca acarreó un desastre no sólo ecológico sino ecológico cultural (Fábregas 2004), al permitirse y alentarse la tala del bosque tropical que, de dos millones de hectáreas de superficie en 1900, terminó en un "manchón” de sólo trescientas mil hectáreas en la actualidad. En este proceso de corrimiento de la frontera agrícola está uno de los orígenes de la sublevación zapatista del 1 de enero de 1994. En otro aspecto, la construcción de las hidroeléctricas desplazó masivamente a la población campesina, parte de la cual se estableció en la selva, aumentando la tensión y los conflictos por la tierra. El 13\% de todo el territorio de cultivo del Estado de Chiapas fue inundado con las consecuencias ecológicas y ecológicas culturales previsibles. La Comisión Federal de Electricidad (CFE), la agencia del Estado Nacional encargada de la construcción de las hidroeléctricas solicitó a Ángel Palerm la elaboración de un proyecto para la reubicación de la población cuyas tierras serían ahogadas. Ello posibilitó la puesta en operación de una serie de trabajos guiados con el método de la ecología cultural, que describieron las técnicas y las tecnologías de cultivo en las áreas que quedaron 
debajo de las aguas, así como de los procesos de cambio perfiladas en las modificaciones a las ecologías culturales locales. Una buena parte de los resultados de estos proyectos aún no se han publicado, aunque existen los textos que fueron presentados como tesis para obtener el grado de Maestros en Antropología en la Escuela Nacional de Antropología e Historia, como es el caso de Bolívar Hernández o Shoko Dodde. Una antropóloga que fue miembro de esos grupos de trabajo, Virginia Molina Ludy (1973), publicó un libro que es un clásico en el ámbito de la antropología mexicana. Me refiero a Venustiano Carranza. Una urbanización frenada. Con este libro, se demostró la aplicabilidad del método de la ecología cultural en el análisis de situaciones urbanas en México. Las recomendaciones para el diseño de los pueblos, las casas, las huertas, los ámbitos de cultivo de la población desplazada, fueron olímpicamente ignoradas por la CFE, lo que provocó serios conflictos sociales en Chiapas. Aquí está otro de los orígenes de la rebelión zapatista de 1994. Las estrategias de adaptación "mal adaptantes" son cruciales como factores de explicación en el tipo de casos expuestos y señalan la utilidad del enfoque sobre el manejo político de la ecología. En otras palabras, la alteración de ecologías culturales sin entender su dinámica interna, por parte del Estado Nacional, ha resultado en desastres sociales y políticos de los que México aún no logra salir. Ello también ha contribuido a profundizar la desigualdad no sólo social sino en el propio desarrollo regional.

La cuestión de la construcción de las hidroeléctricas en Chiapas renovó la discusión de los planteamientos de Wittfogel acerca de la capacidad del Estado para movilizar a grandes contingentes de trabajadores y modificar las ecologías culturales locales. En el caso chiapaneco, varios de los poblados campesinos fueron obligados a convertirse en pescadores y aprender a manejar una nueva tecnología, que les era ajena, como lo es la pesca en embalses. Una amplia superficie de la depresión central de Chiapas fue severamente alterada en su ecología cultural por razones políticas. Las ancestrales ecologías culturales asociadas al manejo del maíz y al cultivo del arroz, desaparecieron, para dar lugar a los lagos artificiales en cuyos fondos yacen los poblados con sus panteones y sus iglesias. Este es un ejemplo clásico de ecología cultural política porque las adaptaciones se diseñan al margen de los intereses de los productores directos a quienes se desplazó argumentándose el "interés nacional". Tan "nacional" es ese interés que en pleno 2008 el territorio de Chiapas aún no está electrificado (falta alrededor de un 35\%) y los apagones son frecuentes incluso en las principales ciudades. Las hidroeléctricas crearon una ecología cultural alienada de la sociedad local.

El grupo de estudio dirigido por Ángel Palerm en Chiapas observó la inminencia de la catástrofe, al establecerse una ecología cultural disgrega- 
da. Por mala fortuna no conocemos con precisión la propia opinión de Palerm que es posible descanse en el fondo de algún cajón burocrático en la CFE o haya sido pasto de los roedores. Pero como el ejemplo chiapaneco pueden citarse otros, como lo ocurrido en la Chontalpa, región de Tabasco, en la que el Estado Nacional irrumpió destruyendo una de las ecologías culturales mejor equilibradas del país, exterminando de paso al Pueblo Chontal, creando una región desolada. En estos ejemplos, como en los casos estudiados por Brigitte Boehm (2005) en la región del Lago de Chapala, está la demostración de que la ecología cultural al igual que la economía, es diseñada y manejada políticamente en el contexto de sociedades profundamente desiguales. Es el manejo interesado de la ecología cultural lo que provoca en la actualidad los problemas de mayor gravedad que afronta el Mundo. Las catástrofes que ya están y las que vienen no solo conllevan el arrasamiento de la naturaleza, sino de Culturas enteradas asociadas a las ecologías culturales que son eliminadas. Ante esos escenarios, una antropología que desvele los procesos mal adaptantes, es de primera importancia. Ello se logra elaborar a través de los análisis de las ecologías culturales políticas y sus relaciones con el Poder y sus instituciones.

\section{BiBLIOGRAFÍA CITADA}

Aguirre Beltrán, G. 1967. Regiones de Refugio. El desarrollo de la comunidad y el proceso dominical en mestizoamérica. México: Fondo de Cultura Económica.

Boëhm de Lameiras, B. 1986. La formación del Estado en el México Prehispánico. Zamora: El Colegio de Michoacán.

Boehm, B. 2003. "Buscando hacer ciencia social. La antropología y la ecología cultural". Relaciones. Estudios de Historia y Sociedad 102 (XXVI): 63-128.

Boehm, B. 2005. "Agua, tecnología y sociedad en la cuenca Lerma-Chapala". Nueva Antropología 64 (XIX): 99-130.

Cohen, Y. A. 1974. Man in adaptation. The cultural present. Chicago: Aldine Publishing House.

Fábregas, A. 1986. La formación histórica de una región: Los Altos de Jalisco. México: CIESAS.

Fábregas, A. 2003. Reflexiones desde la Tierra Nómada. México: El Colegio de San Luis.

Fábregas, A. 2004. "Chiapas. La cuna del conflicto”, en L. Díaz G. Viana (coord.), El nuevo orden del caos: 263-271. Madrid: CSIC

Gamio, M. 1922. La población del Valle de Teotihuacan. El medio en que se ha desarrollado, su evolución étnica y social. Iniciativas para procurar su mejoramiento. México: Dirección de Antropología. 3 vols.

Gómez Sahagún, L. 1992. San Miguel Tlaixpan. Cultivo tradicional de la flor. México: Universidad Iberoamericana.

Krader, L. 1972. The etnological notebooks of Karl Marx. Assen: Van Gorcum. [Hay ed. en castellano: 1988. Cuadernos de Notas Etnológicas. Edición, introducción y notas de Lawrence Krader. Madrid: Siglo XXI]. 
Krader, L. 1973. "Marx as Ethnologist", en Transactions of the New York Academy of Sciences. Series II, Volúmen 35- 4: 304-314. Abril 1973.

Krader, L. 1975. The Asiatic Mode of Production. Assen: Van Gorcum.

Manners, R. y D. Kaplan (eds.). Theory in Anthropology. Chicago: Aldine Publishing Company.

Marx, K. 1968a. "The Bristish Rule in India", en S. Avineri (ed.), Karl Marx on Colonialism and Modernization: 88-95. Nueva York: Anchor Books, (Publicado originalmente en el New York Daily Tribune el 1 de Julio de 1853).

Marx, K. 1968b. "The future results if British Rule in India", en S. Avineri (ed.), Karl Marx on Colonialism and Modernization: 132-139 Nueva York: Anchor Books (Publicado originalmente en el New York Daily Tribune el 8 de agosto de 1853).

Molina Ludy, V. 1973. Venustiano Carranza. Una urbanización frenada. México: SEPINAH.

Murra, J. V. 1975. Formaciones económicas y políticas del mundo andino. Lima: Instituto de Estudios Peruanos.

Palerm, Á. 1952. "La distribución del regadío en Mesoamérica". Ciencias Sociales. Washington, D.C., Unión Panamericana, Departamento de Asuntos Culturales, Oficina de Ciencias Sociales, Vol. V, Número 25, 1954: 2-15.

Palerm, Á. 1955. "La base agrícola de la civilización urbana en Mesoamérica", en J. H. Steward (ed.), Las civilizaciones antiguas del viejo mundo y de América: Symposium sobre las civilizaciones de regadio. (Estudios Monográficos, 1): 29-44. Washington, D.C.: Pan American Union.

Palerm, Á. 1967. Introducción a la Teoría Etnológica. México: Universidad Iberoamericana.

Palerm, Á. 1980. Antropología y marxismo. México: CIS-INAH-Ed. Nueva Imagen.

Palerm, Á. 1990. México prehispánico. Evolución ecológica del valle de México. México: CONACULTA.

Palerm, A. y E. Wolf. 1954-1955. "El desarrollo del área clave del imperio texcocano". Revista Mexicana de Estudios Antropológicos 14: 337-349.

Palerm, A. y E. Wolf. 1972. Agricultura y civilización en Mesoamérica. México: SepSetentas.

Sahlins, M. D. y E. R. Service. 1960. Evolution an Culture. Ann Arbor: University of Michigan Press.

Tomé, P. y A. Fábregas. 1999. Entre Mundos. Relaciones interculturales entre México y España. Zapopan, Jal.: El Colegio de Jalisco.

Steward, J. 1955. Theory of culture change. Urbana: University of Illinois Press.

Steward, J. (ed.). 1956. The people of Puerto Rico. Urbana: University of Illinois Press.

Vivó Escoto, J. A. 1951. Integración de Chiapas a la Nación Mexicana. Tesis para obtener el título de Etnólogo y el grado de Maestro en Ciencias Antropológicas presentada en la Escuela Nacional de Antropología e Historia, México.

Wittfogel, K. A. 1957. Oriental Despotism. New Haven: Yale University Press.

Wolf, E. 1959. Son of the shaking earth. Chicago: University of Chicago Press.

Fecha de recepción: 6 de octubre de 2008

Fecha de aceptación: 5 de febrero de 2009

RDTP, vol. LXIV, n. ${ }^{\circ}$ 1, pp. 167-176, enero-junio 2009, ISSN: 0034-7981, eISSN: 1988-8457, doi: 10.3989/rdtp.2009.015 